\title{
Holographic superconductor/insulator transition at zero temperature
}

\author{
Tatsuma Nishioka, ${ }^{a, b}$ Shinsei Ryu $^{c}$ and Tadashi Takayanagi ${ }^{b}$ \\ ${ }^{a}$ Department of Physics, Kyoto University, \\ Kyoto, 606-8502, Japan \\ ${ }^{b}$ Institute for the Physics and Mathematics of the Universe, \\ University of Tokyo, Kashiwa, Chiba 277-8582, Japan \\ ${ }^{c}$ Department of Physics, University of California, \\ Berkeley, CA 94720, U.S.A. \\ E-mail: nishioka@gauge.scphys.kyoto-u.ac.jp, sryu@berkeley.edu, \\ tadashi.takayanagi@ipmu.jp
}

ABSTRACT: We analyze the five-dimensional AdS gravity coupled to a gauge field and a charged scalar field. Under a Scherk-Schwarz compactification, we show that the system undergoes a superconductor/insulator transition at zero temperature in $2+1$ dimensions as we change the chemical potential. By taking into account a confinement/deconfinement transition, the phase diagram turns out to have a rich structure. We will observe that it has a similarity with the RVB (resonating valence bond) approach to high- $T_{c}$ superconductors via an emergent gauge symmetry.

KEYwords: Black Holes in String Theory, AdS-CFT Correspondence

ArXiv EPRINT: 0911.0962 


\section{Contents}

1 Introduction $\quad 1$

2 Holographic superconductor/insulator transition at zero temperature $\quad 3$

2.1 Superconductor/Insulator in $\mathrm{AdS}_{5}$ soliton 3

$\begin{array}{lll}2.2 \text { Conductivity } & 6\end{array}$

$\begin{array}{llll}3 & \mathrm{AdS}_{5} & \text { charged black hole and superconductor } & 7\end{array}$

$\begin{array}{lll}3.1 & \text { Conductivity in metallic phase } & 8\end{array}$

3.2 Holographic superconductor in $\mathrm{AdS}_{5}$ black hole 8

4 Phase structure $\quad 9$

4.1 Phase transition between $\mathrm{AdS}_{5}$ soliton and $\mathrm{AdS}_{5}$ black hole 10

$\begin{array}{lll}4.2 & \text { Phase diagram } & 11\end{array}$

$\begin{array}{lll}4.3 & \text { String theory embedding } & 11\end{array}$

4.4 The $t$ - $J$ model and the slave boson approach in RVB theory 12

4.5 Comparison between the holographic calculations and the slave boson approach 15

$\begin{array}{lll}5 & \text { Conclusions } & 17\end{array}$

$\begin{array}{lr}\text { A Schrödinger potential description } & 18\end{array}$

$\begin{array}{lll}\text { A.1 AdS soliton } & 18\end{array}$

$\begin{array}{lll}\text { A.2 } & \text { AdS black hole } & 20\end{array}$

\section{Introduction}

The AdS/CFT correspondence [1] is a very powerful method to analyze the phase structures and phase transitions in strongly coupled quantum many-body systems. This is because these complicated quantum problems are equivalently mapped to the study of their gravity duals, each of which is often described by a classical Einstein gravity coupled to various matter fields.

A rich phase diagram can appear in quantum many-body systems when there are many competing and mutually frustrating interactions. For example, one of the richest and most interesting phase structures has been known to appear in strongly correlated materials, such as the cuprate high- $T_{c}$ superconductors [2] (see the figure 10 in the main text), and layered organic conductors. In the phase diagram of high- $T_{c}$, an insulator phase with the antiferromagnetic order (called the Mott insulator) is located close to the superconductor phase. This implies that the strong Coulomb repulsive interaction is behind the pairing mechanism of the superconductivity: It is highly likely that superconductivity is not driven by a conventional mechanism due to simple attractive forces. Instead the superconductivity 
emerges as the best compromise among many competing ground states. Adjacent to the Mott insulator and superconductor phases, there appear the pseudo gap and strange metal phases, where transport properties are highly unusual. As we increase doping $x$ beyond the superconductivity phase, there is the fermi-liquid phase.

The purpose of this paper is to study such a complex competition of different quantum ground states in a simple setup of AdS/CFT. Following the idea of the holographic superconductor [3-11], we consider the Einstein gravity coupled to a U(1) gauge field and a charged scalar field. The U(1) gauge field is interpreted as an R-charge and the scalar field is dual to an R-charged operator. An important difference from the earlier works is that in our setup the holographic superconductor is put in the five-dimensional AdS soliton background $[12,13]$. As we compactify one of the space directions in this asymptotic AdS spacetime, the AdS soliton is dual to a Scherk-Schwarz compactification of a fourdimensional conformal gauge theory, such as the $\mathcal{N}=4$ super Yang-Mills theory. The dual field theory is thus $(2+1)$-dimensional as is so in the high- $T_{c}$ superconductors.

In the holographic superconductors, there is a finite temperature superconductor-metal transition. On the other hand, the AdS soliton background decays into AdS black hole via a Hawking-Page transition [14], which is dual to the confinement/deconfinement transition [12]. One of our main findings is that at zero temperature, as we change the chemical potential, there is an intervening phase between the holographic superconductor phase and the confining phase, which we call the AdS soliton superconductor phase (refer to figure 9 in the main text). While this phase is similar to the holographic superconductor phase (labeled as the AdS BH superconductor phase in figure 9) in the sense that it is the phase where the charged scalar condenses, this phase has a much larger gap. The confinement phase (labeled as the AdS soliton in figure 9) is a phase with a mass gap and an analogue of an insulator in electronic systems. Our setup thus realizes, as we change the chemical potential, an insulator-to-superconductor quantum phase transition.

We will compare these findings with the RVB (resonating valence bond) approach to high- $T_{c}$ superconductors [15]. The RVB theory starts by taking into account the effects of the strong Coulomb interaction which is most operative near zero doping, $x \sim 0$. The strong Coulomb repulsion forbids any site to be occupied by more than one electron, and thus serves effectively as a constraint on the physical Hilbert space. This constraint can be written as a gauge constraint, and therefore physics of high- $T_{c}$ superconductors near $x \sim 0$ can be written in the language of an $\mathrm{SU}(2) / \mathrm{U}(1) / \mathbb{Z}_{2}$ gauge theory (the gauge group depends on, at the level of the mean field theory, the choice of a saddle point.) The quasiparticles charged under the gauge field is not an electron, but an emergent entity which carries a fraction of the quantum number of electrons. The abnormal phases in high- $T_{c}$, i.e., the phases which are not well-described in terms of electrons, can then be potentially described in terms of these emergent particles when they are deconfined, whereas confinement thereof gives rise to conventional phases. The high- $T_{c}$ superconductor thus provides a venue where there is a complex interplay between confinement/deconfinement physics and superconductivity in $(2+1)$ dimensions.

This paper is organized as follows: in section 2, we will show that an superconductor/insulator phase transition will occur in the AdS soliton background. In section 3, we 
will analyze the superconductor phase in the $\mathrm{AdS}_{5}$ black hole. In section 4, we will consider the phase diagram of our system and discuss its relation to the RVB approach to the high- $T_{c}$ superconductors. In section 5 we will summarize conclusions.

\section{Holographic superconductor/insulator transition at zero temperature}

Let us begin with the five-dimensional Einstein-Maxwell-scalar theory:

$$
S=\int d^{5} x \sqrt{-g}\left(R+\frac{12}{L^{2}}-\frac{1}{4} F^{\mu \nu} F_{\mu \nu}-\left|\nabla_{\mu} \Psi-i q A_{\mu} \Psi\right|^{2}-m^{2}|\Psi|^{2}\right) .
$$

Notice that almost the same system appears in the $\operatorname{AdS}_{5} \times \mathrm{S}^{5}$ compactification dual to $\mathcal{N}=4$ super Yang-Mills. ${ }^{1}$ There, the $A_{\mu}$ and $\Psi$ can be dual to a R-charge current and a R-charged operator with charge $q$, though we will not further pursuit a string theory interpretation in this paper. In section 4.3 , we will show that we can consistently embed our phase transition into the $\mathcal{N}=1$ superconformal field theory dual to $\operatorname{AdS}_{5} \times T^{1,1}$.

In this theory, our setup is defined by an asymptotically AdS spacetime which approaches to $R^{1,2} \times S^{1}$ near the boundary. In this section we only consider the case of zero temperature. We impose the anti-periodic boundary condition for fermions (i.e. ScherkSchwarz boundary condition) in the $S^{1}$ direction. When the gauge field is vanishing, the most stable configuration which satisfies this property is known as the AdS soliton [12, 13] given by the metric

$$
\begin{aligned}
& d s^{2}=L^{2} \frac{d r^{2}}{f(r)}+r^{2}\left(-d t^{2}+d x^{2}+d y^{2}\right)+f(r) d \chi^{2}, \\
& f(r)=r^{2}-\frac{r_{0}^{4}}{r^{2}} .
\end{aligned}
$$

This can be obtained by double Wick rotating the AdS Schwarzschild black hole. In order to have a smooth geometry we need to impose the periodicity $\chi \sim \chi+\frac{\pi L}{r_{0}}$ for the ScherkSchwarz circle. If we extract the coordinates $(r, \chi)$, the geometry looks like a cigar, whose tip is given by $r=r_{0}$.

Now we take into account the coupling of this system to the gauge field. We can easily find a simple solution with the constant gauge potential $A_{t}=\mu .^{2}$ Notice that as opposed to the AdS black holes which require $A_{t}=0$ at the horizon, the boundary condition is chosen so that the gauge field is non-singular at the tip.

\subsection{Superconductor/Insulator in $\mathbf{A d S}_{5}$ soliton}

In the presence of charged scalar field as in (2.1), the phase structure can be more nontrivial as the scalar field can condense. We would like to analyze this below assuming that

\footnotetext{
${ }^{1}$ Though in many string theory setups there exist Chern-Simons terms, we will not consider it in this paper as they do not change our solutions. Recently a new possibility of instability in the presence of Chern-Simons terms has been found in [16].

${ }^{2}$ In general, it is possible that we have other solutions which look more non-trivial. However, we do not discuss it because in this paper we are always working within the approximation where we can ignore the backreaction of gauge field and scalar to the metric.
} 
the backreaction of the gauge field and scalar to the metric (2.2) is negligible. We focus on the solutions depending on only radial coordinate as follows:

$$
A=\Phi(r) d t, \quad \Psi=\Psi(r) .
$$

Moreover, we take $\Psi$ to be real due to the Maxwell equation. We can also set $L=1$ and $r_{0}=1$ without losing generality. At zero temperature the system depends on the parameters $\left(q, \mu, m^{2}\right)$.

The equations of motion are

$$
\begin{aligned}
\Psi^{\prime \prime}+\left(\frac{f^{\prime}}{f}+\frac{3}{r}\right) \Psi^{\prime}+\left(-\frac{m^{2}}{f}+\frac{q^{2} \Phi^{2}}{r^{2} f}\right) \Psi & =0, \\
\Phi^{\prime \prime}+\left(\frac{f^{\prime}}{f}+\frac{1}{r}\right) \Phi^{\prime}-\frac{2 q^{2} \Psi^{2}}{f} \Phi & =0 .
\end{aligned}
$$

We have to impose the boundary condition at the tip $r=r_{0}$ and the boundary $r=\infty$ to solve the above equations. We will concentrate on the case where $m^{2}$ is given by $m^{2}=-\frac{15}{4}$ which satisfies the BF bound $m^{2}>-4$. Though this is just for simplicity, we can still extend our analysis to general values of $m^{2}$. Near the boundary, the solutions behave as

$$
\begin{aligned}
& \Psi=\frac{\Psi^{(1)}}{r^{3 / 2}}+\frac{\Psi^{(2)}}{r^{5 / 2}}+\ldots, \\
& \Phi=\mu-\frac{\rho}{r^{2}}+\ldots .
\end{aligned}
$$

At this value of $m^{2}$, there are two alternative descriptions since both terms, proportional to $\Psi^{(1)}$ and $\Psi^{(2)}$, respectively, become normalizable. We defined the corresponding dual operators by $\mathcal{O}_{1}$ and $\mathcal{O}_{2}$ whose conformal dimensions are give by $\Delta=3 / 2$ and $\Delta=5 / 2$, respectively.

On the other hand, at the tip these behave as

$$
\begin{aligned}
& \Psi=a+b \log \left(r-r_{0}\right)+c\left(r-r_{0}\right)+\ldots \\
& \Phi=A+B \log \left(r-r_{0}\right)+C\left(r-r_{0}\right)+\ldots .
\end{aligned}
$$

Therefore, we impose the Neumann-like boundary condition $b=B=0$ to take every physical quantities finite.

In (2.7), the constants $\mu$ and $\rho$ are holographically dual to the chemical potential (gauge potential) and the charge density, respectively.

As is clear from (2.5), the equations of motion have the scaling symmetry $(\Phi, \Psi, \mu, q) \rightarrow$ $(\lambda \Phi, \lambda \Psi, \lambda \mu, q / \lambda)$. By taking $\lambda \ll 1$ (the probe limit), we can indeed neglect the back reactions of $\Phi$ and $\Psi$ to the metric as in the standard holographic superconductors $[3,8]$. In other words, this probe approximation is justified when $\mu \ll 1$ and $q \gg 1$ with $\mu q$ kept finite. Once we take this probe limit, the functional form of physical quantities up to the overall scaling only depend on the combination $\mu q$ in this probe limit. Thus below we will simply set $q=1$. 

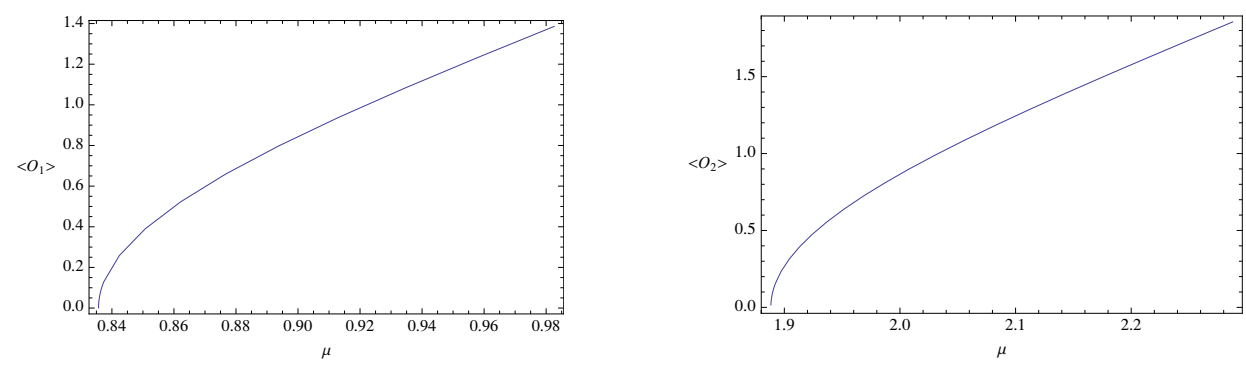

Figure 1. The condensations of the scalar operators $\left\langle\mathcal{O}_{1}\right\rangle$ (left) and $\left\langle\mathcal{O}_{2}\right\rangle$ (right).
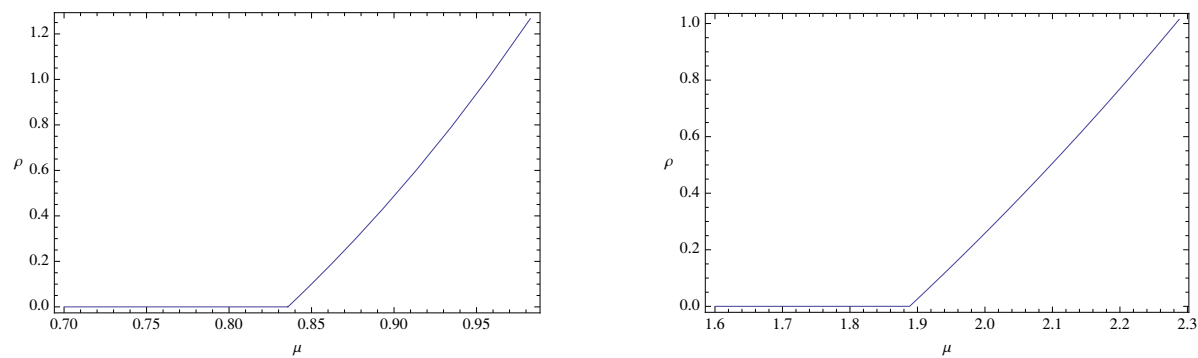

Figure 2. The charge density $\rho$ plotted as a function of $\mu$ when $\left\langle\mathcal{O}_{1}\right\rangle \neq 0$ (left) and $\left\langle\mathcal{O}_{2}\right\rangle \neq 0$ (right). Its derivative jumps at the phase transition point.

In the AdS/CFT dictionary, the asymptotic behavior of $\Phi$ gives the chemical potential $\mu$ and charge density $\rho$ in the dual field theory. The scalar operator $\mathcal{O}$ coupled to the scalar field $\Psi$ is (up to a normalization)

$$
\left\langle\mathcal{O}_{i}\right\rangle=\Psi^{(i)}, \quad i=1,2
$$

with the boundary condition $\epsilon_{i j} \Psi^{(j)}=0$. We can plot $\left\langle\mathcal{O}_{i}\right\rangle$ with respect to the chemical potential $\mu$ by numerical calculations and find that the condensation occurs if $\mu>\mu_{i}$ ( $\mu_{1}=0.84$ and $\mu_{2}=1.88$ ) as in figure 1 . Notice that for any values of $q$, the probe approximation offers us exact critical values $\mu_{1,2}$ and behaviors near the the critical points, where the back reactions are highly suppressed.

In this way, we find a phase transition triggered by the scalar field condensation in the AdS soliton background. We also find that it is a second order phase transition as is clear if we plot the charge density $\rho(=\partial \Omega / \partial \mu)$ as a function of $\mu$ as shown in figure 2 . When $\mu$ is small, the system is described by the AdS soliton solution itself. This is interpreted as the insulator phase as this system has a mass gap, which is due to the confinement in the $(2+1)$-dimensional gauge theory viewpoint via the Scherk-Schwarz compactification. On the other hand, for a larger $\mu$, it undergoes a phase transition and is expected to reach a superconductor (or superfluid) phase. Thus we can regard this as the holographic realization of superconductor/insulator transition.

The mechanism of this transition is similar to the standard holographic superconductors $[3,8]$, but is different in that our phase transition occurs even at strictly zero temperature. We also give an explanation of the transition by relating it to the Schrödinger problem in the appendix A. 
If we impose the periodic boundary condition instead of the anti-periodic one for fermions, the story will change largely because the AdS soliton phase is not available and the confinement will not occur at zero temperature. In this case, we need to consider a zero temperature limit of holographic superconductor in AdS black holes, which has been analyzed in [17-20]. We will not discuss this case in this paper.

So far we only consider the zero temperature system. At finite temperature $T$, it is described by compactifying the Euclidean time direction. When $T$ is greater than a certain value $T_{c}$, a phase transition like confinement/deconfinement transition occurs as we will see later. Thus our argument of phase transition can equally be applied to the finite temperature case as long as we have $T<T_{c}$ and we can ignore the back reaction.

\subsection{Conductivity}

We can holographically calculate the conductivity $\sigma(\omega)$ by solving the equation of motion of $A_{x} \propto e^{-i \omega t}$ :

$$
A_{x}^{\prime \prime}+\left(\frac{f^{\prime}}{f}+\frac{1}{r}\right) A_{x}^{\prime}+\left(\frac{\omega^{2}}{r^{2} f}-\frac{2 q^{2} \Psi^{2}}{f}\right) A_{x}=0,
$$

by requiring the Neumann boundary condition at the tip $r=r_{0}$ as in (2.8). Looking at the asymptotic behavior near the boundary $r \rightarrow \infty$

$$
A_{x}=A_{x}^{(0)}+\frac{A_{x}^{(1)}}{r^{2}}+\frac{A_{x}^{(0)} \omega^{2}}{2} \frac{\log \Lambda r}{r^{2}}+\cdots,
$$

the holographic conductivity is found as follows [7]

$$
\sigma(\omega)=\frac{-2 i A_{x}^{(1)}}{\omega A_{x}^{(0)}}+\frac{i \omega}{2} .
$$

First let us consider the AdS soliton background (2.2) without the scalar condensation. Since there is no horizon, the equation of motion with the boundary condition at the tip requires that $\frac{A_{x}^{(1)}}{A_{x}^{(0)}}$ is real. Therefore the real part of the conductivity vanishes. This means that there is no dissipation and is consistent with the absence of horizon. The imaginary part of $\sigma(\omega)$ is plotted in the left graph of figure 3. In this way, we can identify the AdS soliton with an insulator. We also notice there are poles periodically at the points where $A_{x}^{(0)}$ vanishes. These correspond to normalized modes dual to vector operators. In the paper [21], this setup has been used to realize the fractional quantum Hall effect by adding D7-branes wrapped on $S^{5}$, which is a basic example of topological insulators (see [22] for its back reacted supergravity solution). On the other hand, if we calculate $\sigma(\omega)$ for the AdS soliton with the scalar condensation by taking $\mu>\mu_{1,2}$, then the imaginary part of $\sigma(\omega)$ is obtained as in the right graph of figure 3. When $\omega$ is large, the behavior looks very similar to the one for the AdS soliton without the scalar condensation. However, in the present case, we can observe a pole even at $\omega=0$. The Kramers-Kronig relation

$$
\operatorname{Im} \sigma(\omega)=\frac{1}{\pi} P \int_{-\infty}^{\infty} d \omega^{\prime} \frac{\operatorname{Re} \sigma\left(\omega^{\prime}\right)}{\omega-\omega^{\prime}} .
$$



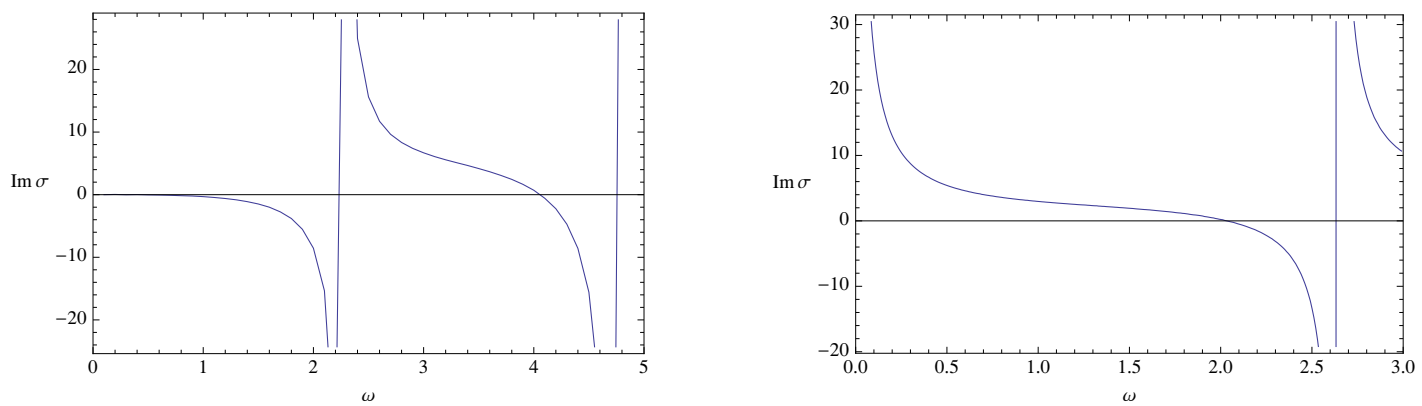

Figure 3. The imaginary part of the conductivity for the AdS soliton without a scalar condensation $\left\langle\mathcal{O}_{1,2}\right\rangle=0$ (left) and with a scalar condensation $\left\langle\mathcal{O}_{1}\right\rangle \neq 0$. We employed the background with $\rho=0.0094$ and $\mu=0.84$ in the right graph.

argues that $\operatorname{Re} \sigma(\omega)$ has a delta functional support. Thus this should be identified with the superconductivity. Defining the superfluid density $n_{s}$ by $\operatorname{Im} \sigma(\omega) \sim \frac{n_{s}}{\omega}$ in the $\omega \rightarrow 0$ limit, we obtain the behavior $n_{s} \sim 20 \mu_{1}\left(\mu-\mu_{1}\right)$ and $n_{s} \sim 1.2 \mu_{2}\left(\mu-\mu_{2}\right)$ for each case near the transition.

\section{$3 \quad \mathrm{AdS}_{5}$ charged black hole and superconductor}

At high temperature, we expect that the AdS soliton background will decay into the AdS black hole via a Hawking-Page transition, which is dual to the confinement/deconfinement transition [12]. It is well expected that this should be true even in the presence of scalar condensations. Therefore, here we want to analyze the properties of five-dimensional charged AdS black hole solutions and their superconductor phase transition. We start with the Einstein-Maxwell theory given by (2.1) by setting $\Psi=0$. The equations of motion look like

$$
\begin{aligned}
\partial_{\mu}\left(\sqrt{-g} g^{\mu \nu} F_{\nu}^{\rho}\right) & =0 \\
R_{\mu \nu}-\frac{R}{2} g_{\mu \nu}-\frac{6}{L^{2}} g_{\mu \nu} & =\frac{1}{2}\left(F_{\mu \sigma} F_{\nu}^{\sigma}-\frac{1}{4} g_{\mu \nu} F_{\rho \lambda} F^{\rho \lambda}\right) .
\end{aligned}
$$

It is straightforward to obtain the the following solution (see e.g. [10])

$$
\begin{aligned}
d s^{2} & =-f(r) d t^{2}+L^{2} f(r)^{-1} d r^{2}+r^{2}\left(d x^{2}+d y^{2}+d z^{2}\right), \\
A_{t} & =\mu\left(1-\frac{r_{+}^{2}}{r^{2}}\right)
\end{aligned}
$$

with the function $f(r)$ given by

$$
f(r)=r^{2}\left(1-\left(b^{2}+1\right) \frac{r_{+}^{4}}{r^{4}}+b^{2} \frac{r_{+}^{6}}{r^{6}}\right)
$$

where we defined $b^{2}=\frac{\mu^{2}}{3 r_{+}^{2}}$. The temperature of this black hole is given by $T=\frac{r_{+}}{2 \pi L}\left(2-b^{2}\right)$. 

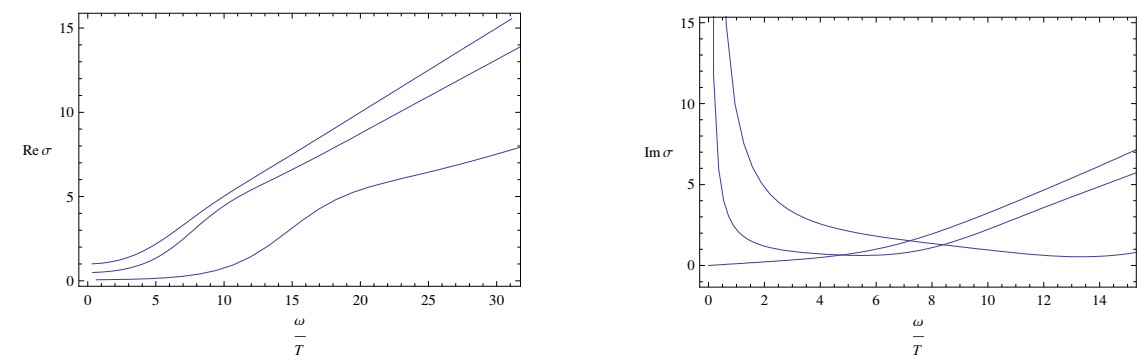

Figure 4. The real and imaginary parts of the conductivity in $\mathrm{AdS}_{5}$ charged black hole. Three curves correspond to $b=0,0.5$ and $b=1$, respectively in the unit $r_{+}=L=1$. When we fixed $\omega / T$, the real part of the conductivity decreases as $b$ becomes large, while the imaginary part increases.

\subsection{Conductivity in metallic phase}

It is also interesting to calculate the conductivity in this background. In the $\mathrm{AdS}_{4}$ black hole background, this has been done in [10]. We assume the fluctuations of $A_{x}$ and $g_{t x}$ have the time dependence $e^{-i \omega t}$. We will set $L=r_{+}=1$ as before.

The Einstein equation for $(x, r)$ component leads to

$$
g_{t x}^{\prime}-\frac{2}{r} g_{t x}-\frac{2 \mu}{r^{3}} A_{x}=0,
$$

and the equation of motion for $A_{x}$ reads

$$
\left(r f(r) A_{x}^{\prime}\right)^{\prime}+\frac{\omega^{2} r}{f(r)} A_{x}+2 \mu r_{+}^{2}\left(\frac{g_{t x}}{r^{2}}\right)^{\prime}=0 .
$$

By combining (3.6) and (3.7), we obtain

$$
\left(r f(r) A_{x}^{\prime}\right)^{\prime}+\left(-\frac{12 b^{2}}{r^{5}}+\frac{\omega^{2} r}{f(r)}\right) A_{x}=0 .
$$

The real and imaginary parts of the conductivity are plotted in figure 4 . The pole of $\operatorname{Im} \sigma(\omega)$ at $\omega=0$ for non-zero values of $\mu$ shows a delta functional contribution to $\operatorname{Re} \sigma(\omega)$ via the Kramers-Kronig relation, which should be smoothed by actual impurities [8, 10].

\subsection{Holographic superconductor in $\mathrm{AdS}_{5}$ black hole}

In the system in (2.1), we can write down the equations of motion for the gauge potential $\Phi$ and the scalar field $\Psi$, which only take real values, as follows:

$$
\begin{aligned}
\Psi^{\prime \prime}+\left(\frac{f^{\prime}}{f}+\frac{3}{r}\right) \Psi^{\prime}+\left(\frac{q^{2} \Phi^{2}}{f^{2}}-\frac{m^{2}}{f}\right) \Psi & =0, \\
\Phi^{\prime \prime}+\frac{3}{r} \Phi^{\prime}-\frac{2 q^{2} \Psi^{2}}{f} \Phi & =0 .
\end{aligned}
$$

The equation of motion for $A_{x}$ is given by

$$
A_{x}^{\prime \prime}+\left(\frac{f^{\prime}}{f}+\frac{1}{r}\right) A_{x}^{\prime}+\left(\frac{\omega^{2}}{f^{2}}-\frac{2 q^{2} \Psi^{2}}{f}\right) A_{x}=0 .
$$



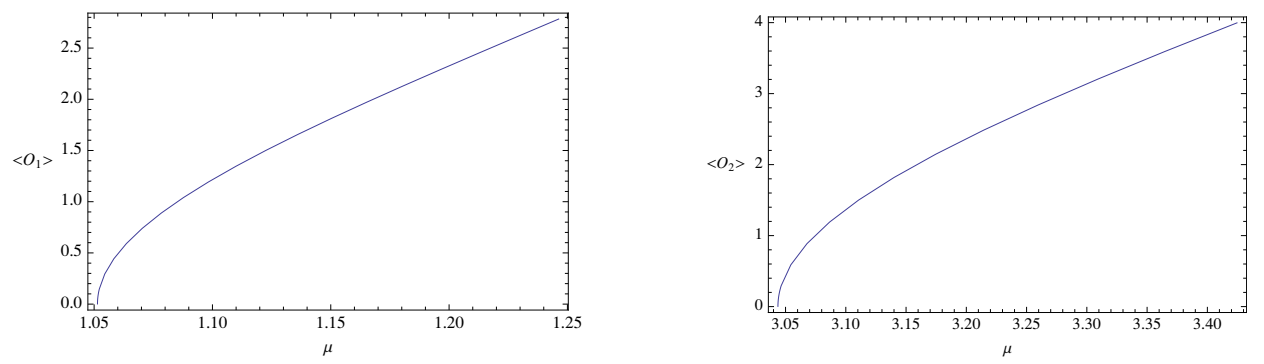

Figure 5. The scalar condense for $\mathcal{O}_{1}$ (left) and $\mathcal{O}_{2}$ (right).
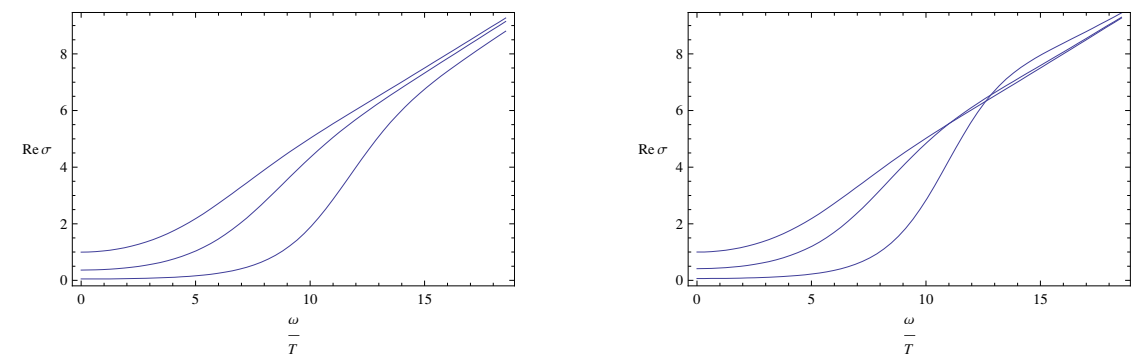

Figure 6. The real part of the conductivity for $\mathcal{O}_{1} \neq 0$ (left) and $\mathcal{O}_{2} \neq 0$ (right). When we fixed $\omega / T$, the real part of the conductivity decreases as $\left|\mu-\mu_{c}\right|$ becomes large.

Now we would like to analyze the holographic superconductor phase in this system by numerical calculations. This system has been studied in [7] for different values of $\mathrm{m}^{2}$. Due to the same reason as before, we set $m^{2}=-\frac{15}{4}, L=r_{+}=q=1$ and $b=0$, which corresponds to the temperature $T=\frac{1}{\pi}$. The result showing the scalar field condensation is plotted in figure 5. It shows that the onset of the phase transition into superconductor occurs when $\mu_{1}=1.05$ (for $\mathcal{O}_{1}$ ) and $\mu_{2}=3.04$ (for $\mathcal{O}_{2}$ ).

If we recover $q$ and $T$, then we can conclude that in the probe approximation the superconductor phase appears when $T<\alpha_{i} q \mu$ due to the condensation of $\left\langle\mathcal{O}_{i}\right\rangle$, where $\alpha_{1}=0.30$ and $\alpha_{2}=0.105$. The superfluid density in this case behaves like $n_{s} \sim 40 \mu_{1}\left(\mu-\mu_{1}\right)$ for $\mathcal{O}_{1}$ and $n_{s} \sim 2.0 \mu_{2}\left(\mu-\mu_{2}\right)$ for $\mathcal{O}_{2}$, respectively. ${ }^{3}$

The real and imaginary part of conductivity is plotted in figure 6 and figure 7 .

\section{Phase structure}

So far, we have studied the superconductor transition both in the $\mathrm{AdS}_{5}$ soliton and $\mathrm{AdS}_{5}$ BH. ${ }^{4}$ Here we would like to analyze the phase transition between $\mathrm{AdS}_{5}$ soliton and in $\mathrm{AdS}_{5}$ $\mathrm{BH}$, which is dual to the confinement/deconfinement transition in $2+1$ dimensional super

\footnotetext{
${ }^{3}$ For $\mathcal{O}_{2}$, the temperature is related with the chemical potential like $1-\frac{T}{T_{c}}=1.65\left(\frac{\mu}{\mu_{2}}-1\right)$ around the critical point $\left(T=T_{c}\right.$ and $\left.\mu=\mu_{2}\right)$. One can obtain $n_{s} \sim 100 T_{c}^{2}\left(1-\frac{T}{T_{c}}\right)$ by using these relations. This result is consistent with that obtained in [7].

${ }^{4}$ We can also consider the pure $\mathrm{AdS}_{5}$ spacetime with the Euclidean time and $\chi$ are compactified. However, it has vanishing free energy $\Omega=0$ and is not favored.
} 

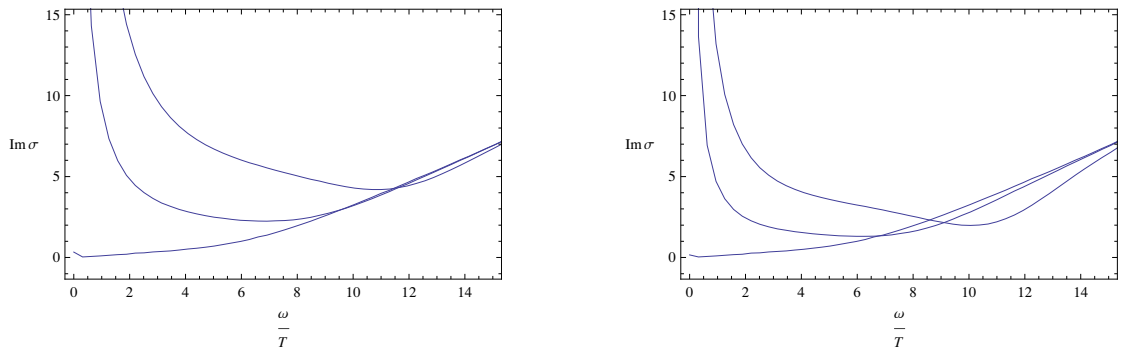

Figure 7. The imaginary part of the conductivity for $\mathcal{O}_{1} \neq 0$ (left) and $\mathcal{O}_{2} \neq 0$ (right). When we fixed $\omega / T$, the imaginary part of the conductivity increases as $\left|\mu-\mu_{c}\right|$ becomes large.

Yang-Mills [12]. After we study this transition, here we would like to capture the phase diagrams of our holographic system.

\subsection{Phase transition between $\mathrm{AdS}_{5}$ soliton and $\mathrm{AdS}_{5}$ black hole}

Let us analyze the phase transition between $\mathrm{AdS}_{5}$ soliton and $\mathrm{AdS}_{5}$ charged black hole in the grand canonical ensemble. The phase boundary is given by the points where the Gibbs Euclidean action $I_{G}$ of $\mathrm{AdS}_{5}$ soliton coincides with that of the $\mathrm{AdS}_{5}$ charged black hole. In the gravity description of $\mathrm{AdS} / \mathrm{CFT}, I_{G}$ is simply related to the classical action (2.1) evaluated for the classical solutions. Here the scalar field is vanishing. In general it is rewritten as follows

$$
I_{G}=\beta \Omega=\beta(E-T S-\mu \rho) .
$$

First consider the $\mathrm{AdS}_{5}$ soliton. In this case, the only contribution to (4.1) is the ADM energy $E$ because $\rho$ and the entropy $S$ are vanishing. It is evaluated as follows in our normalization ${ }^{5}$

$$
\frac{\Omega_{s l}}{V_{2}}=\frac{E}{V_{2}}=-\frac{\pi^{4} L^{3}}{R_{0}^{3}},
$$

where $R_{0}=\frac{\pi L}{r_{0}}$ is the periodicity of $\chi . V_{2}$ denotes the infinite volume of $(x, y)$ plane.

Next we turn to the $\mathrm{AdS}_{5}$ charged black hole. By employing the result in [10], we find in our unit

$$
\frac{\Omega_{b h}}{V_{2}}=-\frac{r_{+}^{4}}{L}\left(1+\frac{\mu^{2}}{3 r_{+}^{2}}\right) R_{0} .
$$

We can now analyze the phase transition from (4.2), (4.3) and the formula of the temperature

$$
T=\frac{r_{+}}{\pi L}\left(1-\frac{\mu^{2}}{6 r_{+}^{2}}\right) .
$$

The smaller values of $\Omega$ among (4.2) and (4.3) are favored. It is easy to see that this phase transition is first order.

For example, when $\mu$ is vanishing $\mu=0$, the phase transition occurs when $T=\frac{1}{R_{0}}$. This is obvious since the transition should be the point where the periodicity of Euclidean time coincides with that of the $\chi$ circle. The AdS black hole and AdS soliton phase correspond

\footnotetext{
${ }^{5}$ We set the Newton constant to $16 \pi G_{N}=1$ in the action $(2.1)$.
} 


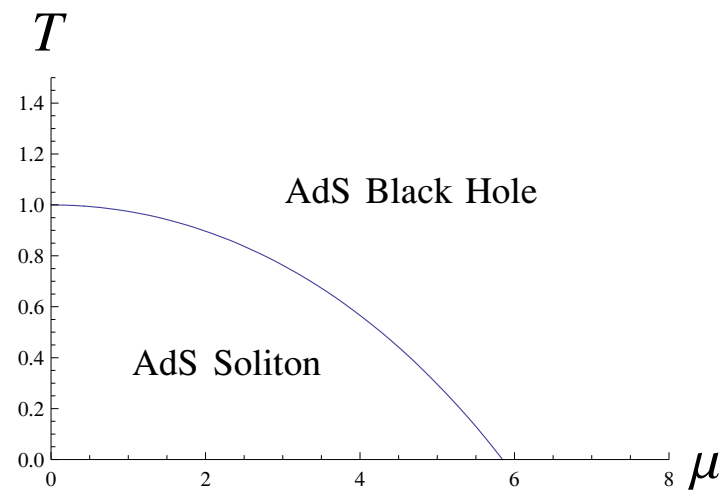

Figure 8. The phase transition between AdS black hole and AdS soliton. The lower region describes the AdS soliton phase, while the other the AdS charged black hole. We set $R_{0}=1$ in this figure.

to $T R_{0}>1$ and $T R_{0}<1$, respectively. On the other hand, when we increase the value of $\mu$ at zero temperature $T=0$, a phase transition occurs at $\mu R_{0}=2^{\frac{1}{2}} 3^{\frac{1}{4}} \pi \simeq 5.85$ from the AdS soliton to AdS charged black hole. We can also analyze the middle regions and find the phase diagram in figure 8.

\subsection{Phase diagram}

Now we are prepared to write down our phase diagram in the probe approximation. We can set $L=1$ without losing generality and also assume $q \gg 1$ so that we can ignore back reactions to the metric. By the coordinate transformation, we fix the periodicity of $\chi$ such as $\chi \sim \chi+\pi$ (corresponding to $r_{0}=1$ in AdS soliton). Thus the independent parameters are $T$ and $\mu$. The probe approximation is good only when $\mu \ll 1$ as it corresponds to the limit $q \rightarrow \infty$ with $\mu q$ kept finite. Therefore we can reliably write down the phase diagram only when $\mu \ll 1$. However, notice that the phase boundary between the AdS soliton and its superconductor is exact even in this approximation and we can capture important structure of the global phase diagram as we will see below.

The basic fact is that in the probe approximation, the free energy difference due to the superconductor phase transition is of order $O\left(q^{-2}\right)$ and thus is much smaller than that of the transition between the $\mathrm{AdS}_{5}$ soliton and $\mathrm{AdS}_{5} \mathrm{BH}$, which is of order $O(1)$. In this way, first we may draw the phase boundary between the $\mathrm{AdS}_{5}$ soliton and $\mathrm{AdS}_{5} \mathrm{BH}$ and then later we can further take into account the superconductor transition. Finally we obtain the phase diagram as shown in figure 9 . The relative position of the superconductor curve follows from the explicit values $\alpha_{1,2}, \mu_{1,2}$ and $T_{c}=\frac{1}{\pi}$, which separate between various phases as we calculated before by setting $m^{2}=-15 / 4$.

\subsection{String theory embedding}

In an actual string theory background, we can interpret the charge $q$ as the R-charge of a certain operator in its dual superconformal field theory. In general, $q$ can be of order one and then the critical chemical potential $\mu_{c}$ is also of order one. Thus $\mu_{c}$ can be greater 


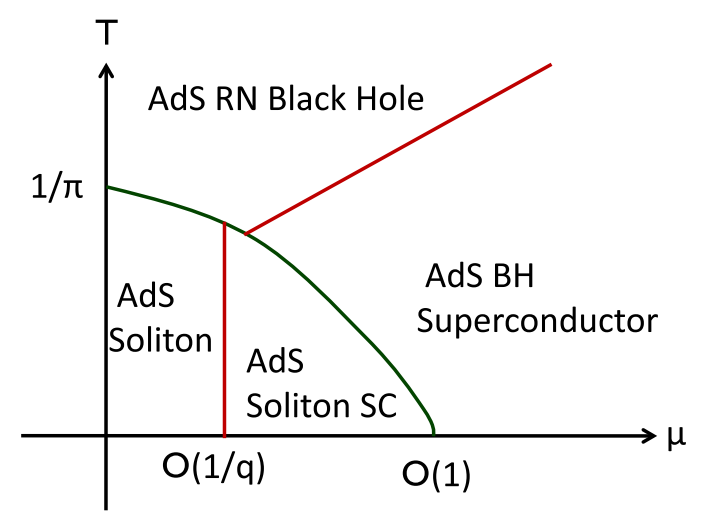

Figure 9. The phase diagram of AdS soliton and AdS black hole with a charged scalar field obtained in the large $q$ limit.

than the confinement/deconfinement transition point $\mu_{d}=2^{\frac{1}{2}} 3^{\frac{1}{4}} \simeq 1.86$ assuming $r_{0}=1$ (or $R_{0}=\pi$ ). If this happens, the AdS soliton solution should be replaced with the AdS black hole at $\mu=\mu_{c}$ and the superconductor phase transition gets unphysical. To see if we can avoid this problem in an explicit string theory setup, consider the $A d S_{5} \times T^{1,1}$ spacetime dual to a four-dimensional $\mathcal{N}=1$ quiver gauge theory [23]. The R-charge $R$ with the standard normalization such that $\Delta=\frac{3}{2} R$ for chiral operators is proportional to our charge via the relation $q=\frac{\sqrt{3}}{2 L} R$ by employing the analysis of the string theory embedding found in [24]. Let us concentrate on the chiral operators of the form $\operatorname{Tr}\left[A_{i} B_{j}\right]$, which have $\Delta=\frac{3}{2}$ and $R=1$. For this operator, our previous analysis shows that the superconductor phase transition in the AdS soliton background occurs at $\mu_{c} \simeq 0.97$. Notice again that the probe analysis is enough to fix the transition point even if $q$ is not large enough. Since this value $\mu_{c}$ is smaller than $\mu_{d}$, we do not have the problem mentioned before. In this way, we have found an example where we can embed our zero temperature superconductor/insulator phase transition into string theory.

\subsection{The $t-J$ model and the slave boson approach in RVB theory}

In this section, we compare the superconductor/insulator transition found in the holographic calculation with the high- $T_{c}$ cuprates, in particular with the RVB scenario of high- $T_{c}[15]$ (see figure 10 for the schematic phase diagram). At the moment, there is no single theory which is capable of explaining all features of the high- $T_{c}$ cuprates for the entire region of the phase diagram. Our focus here is on the so-called under-doped region (the region of phase diagram close to $x=0$ ), where the RVB scenario of high- $T_{c}$ was proposed. Its applicability to the cuprates, in particular, beyond the under-doped region, has been debated. On the other hand, for the over-doped region (the region of phase diagram $x \gtrsim 0.3$ ), the Fermi-liquid ground state would be a reasonable starting point. The electron correlation effects, in particular, antiferromagnetic spin fluctuations, can then be included perturbatively [25-28].

For an application of the AdS/CFT to high- $T_{c}$, see [10, 29, 30, 34, 35], where in this scenario the anomalous transport in the strange metal phase is attributed to the quantum 

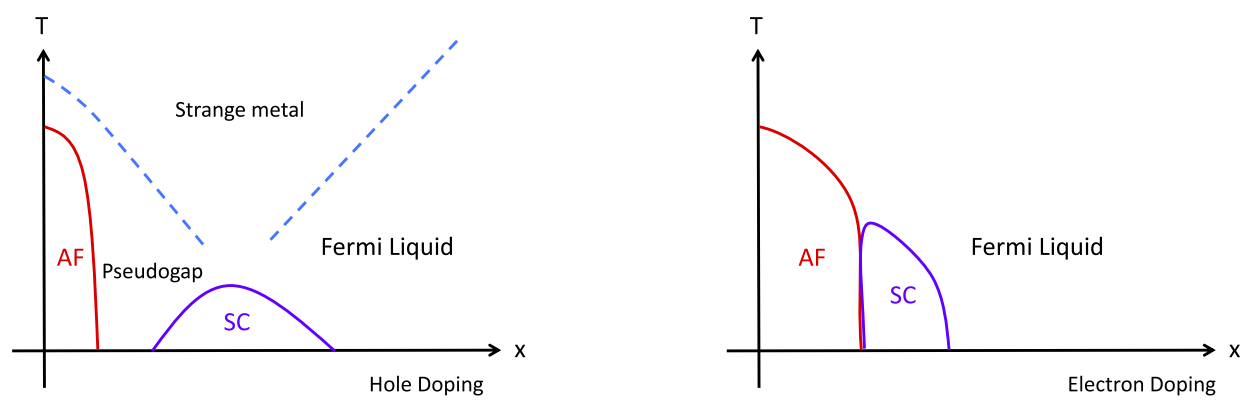

Figure 10. The schematic phase diagrams of the high- $T_{c}$ cuprate superconductors. The left panel is for hole-doped cuprates (e.g. $\mathrm{La}_{2-x} \mathrm{Sr}_{x} \mathrm{CuO}_{4}$ ), while the right is for electron-doped cuprates (e.g. $\mathrm{Nd}_{2-x} \mathrm{Ce}_{x} \mathrm{CuO}_{4}$ ), where $x$ measures the amount of doping. The phase AF denotes the antiferromagnetic phase, which is a Mott insulator; In the pseudo gap region, while it is located above the critical temperature, an energy gap is already observed on the Fermi surface; The metallic region above the superconducting dome near the optimal doping is called the strange metal phase, which shows anomalous transport properties; In the Fermi liquid phase the ground state is adiabatically connected to the free-fermion ground state with a well-defined Fermi surface.

critical transport in the quantum critical regime of the zero temperature quantum critical point. For other approaches to high- $T_{c}$, see $[2,15,25-28]$, and references therein. In the following, we would like to suggest a connection between the AdS/CFT and the high- $T_{c}$ superconductors from a different viewpoint.

The high- $T_{c}$ cuprate at $x=0$ is the antiferromagnetic insulator. By doping holes, we frustrate the antiferromagnetic order and eventually destroy it. Beyond some critical doping $x_{c}$, the superconducting ground state emerges. The physics of the under-doped cuprates is well-described by the $t-J$ model,

$$
H=P\left[-t \sum_{\langle i j\rangle, \sigma} c_{i \sigma}^{\dagger} c_{j \sigma}-\mu \sum_{i} n_{i}+J \sum_{\langle i j\rangle}\left(\boldsymbol{S}_{i} \cdot \boldsymbol{S}_{j}-\frac{1}{4} n_{i} n_{j}\right)\right] P .
$$

Here, $c_{i \sigma}^{\dagger}\left(c_{i \sigma}\right)$ are an electron creation (annihilation) operator at site $i$ on the square lattice with spin $\sigma=\uparrow, \downarrow ; n_{i}=\sum_{\sigma} c_{i \sigma}^{\dagger} c_{i \sigma}$, and $\boldsymbol{S}_{i}=(1 / 2) \sum_{\sigma, \sigma^{\prime}} c_{i \sigma}^{\dagger} \boldsymbol{\sigma}_{\sigma \sigma^{\prime}} c_{i \sigma^{\prime}}$ are the electron number operator, and the spin operator, respectively; $t$ represents the kinetic energy for electron (or hole) hopping and $J$ is the antiferromagnetic exchange; $P$ is the projection operator which removes states with double occupancy at a site $i$ from the physical subspace. The $t-J$ model can be derived from the Hubbard model near half-filling $(x=0)$ by the degenerate perturbation theory, and faithfully captures the physics near $x=0$.

The major obstacle one encounters when trying to solve the $t$ - $J$ model is the constraint (projection) $P$; it is nothing but the strong Coulomb repulsion. The constraint can be analyzed in terms of the so-called slave-boson approach (gauge theory approach); it is a calculational tool representing the idea of the RVB, and for which we make a comparison with our gravity calculations. In the slave-boson (SU(2) slave-boson) approach, we decom- 
pose electron operators into bosonic $h_{i}=\left(b_{i 1}, b_{i 2}\right)$ and fermionic $\psi_{i}=\left(f_{i \uparrow}, f_{i \downarrow}^{\dagger}\right)$ parts as

$$
\begin{aligned}
& c_{i \uparrow}=\frac{1}{\sqrt{2}} h_{i}^{\dagger} \psi_{i}=\frac{1}{\sqrt{2}}\left(b_{1 i}^{\dagger} f_{\uparrow i}+b_{2 i}^{\dagger} f_{\downarrow i}^{\dagger}\right), \\
& c_{i \downarrow}=\frac{1}{\sqrt{2}} h_{i}^{\dagger} \bar{\psi}_{i}=\frac{1}{\sqrt{2}}\left(b_{1 i}^{\dagger} f_{\downarrow i}-b_{2 i}^{\dagger} f_{\uparrow i}^{\dagger}\right) .
\end{aligned}
$$

This splitting of electrons is designed in such a way that it captures the low-energy degrees of freedom in the problem; the bosonic field is called holons and roughly describes the charge degree of freedom of an electron, whereas the fermionic field is called spinons and describes the spin degree of freedom of an electron. When holons and spinons are treated as a free particle, the Hilbert space for them is larger than the original Hilbert space, which is three-dimensional per site. Any physical states |phys $\rangle$ should belong to the physical subspace of the fictitious Hilbert space, and are subjected to the following local constraints:

$$
\left.\left(\psi_{i}^{\dagger} \tau^{l} \psi_{i}+h_{i}^{\dagger} \tau^{l} h_{i}\right) \mid \text { phys }\right\rangle=0, \quad l=1,2,3, \quad \forall i
$$

The constraint can be implemented in the path integral by including a Lagrange multiplier $a_{0}^{l}$. The Heisenberg interaction, which takes on the form of a four fermion interaction, can be decoupled by introducing an auxiliary field $U_{i j}$ defined on a link connecting site $i$ and $j$. The partition function in the imaginary time path-integral is then given by

$$
Z=\int \mathcal{D} \psi \mathcal{D} \psi^{\dagger} \mathcal{D} h^{\dagger} \mathcal{D} h \mathcal{D} a_{0}^{1} \mathcal{D} a_{0}^{2} \mathcal{D} a_{0}^{3} \mathcal{D} U \exp \left(-\int_{0}^{\beta} d \tau L\right)
$$

where the Lagrangian is given by

$$
\begin{aligned}
L= & \frac{3 J}{8} \sum_{\langle i j\rangle} \operatorname{tr}\left(U_{i j}^{\dagger} U_{i j}\right)+\frac{3 J}{8} \sum_{\langle i j\rangle}\left(\psi_{i}^{\dagger} U_{i j} \psi_{j}+\text { h.c. }\right)-\frac{t}{2} \sum_{\langle i j\rangle}\left(\psi_{i}^{\dagger} h_{i} h_{j}^{\dagger} \psi_{j}+\text { h.c. }\right) \\
& +\sum_{i} \psi_{i}^{\dagger}\left(\partial_{\tau}-i a_{0 i}^{l} \tau^{l}\right) \psi_{i}+\sum_{i} h_{i}^{\dagger}\left(\partial_{\tau}-i a_{0 i}^{l} \tau^{l}+\mu\right) h_{i} .
\end{aligned}
$$

Integrating over $a_{0}^{l}$ gives rise to the constraints.

So far we have just rewritten the $t$ - $J$ model. A typical approach to solve this model, within this representation, is then to first look for a mean field, and then include fluctuations around it. The field $a_{0}^{l}$ is interpreted as the time component of a SU(2) gauge field $A_{\mu}^{l}$ and its space components come from the fluctuation of $U_{i j}$.

The mean field phase diagram from the $\mathrm{SU}(2)$ slave boson theory is shown in figure 11 (right). (A similar mean field phase diagram can be drawn from the slightly different formalism of the slave boson theory, the U(1) slave boson theory [figure 11 (left)]). The details of the phase diagram depend on the values of $t$ and $J$, e.g., the $\pi$-flux does not appear for large $t / J$, but the qualitative features do not depend too sensitively on $t / J$.

To go beyond the mean field theory, we need to include fluctuations around a saddle point (mean field). For a given saddle point configuration for $U_{i j}$, the low-energy gauge field fluctuations can be described by either one of $\mathrm{SU}(2), \mathrm{U}(1)$, or $\mathbb{Z}_{2}$ group, since a meanfield configuration $U_{i j}$ can Higgs out partially the $\mathrm{SU}(2)$ gauge fluctuations. While 

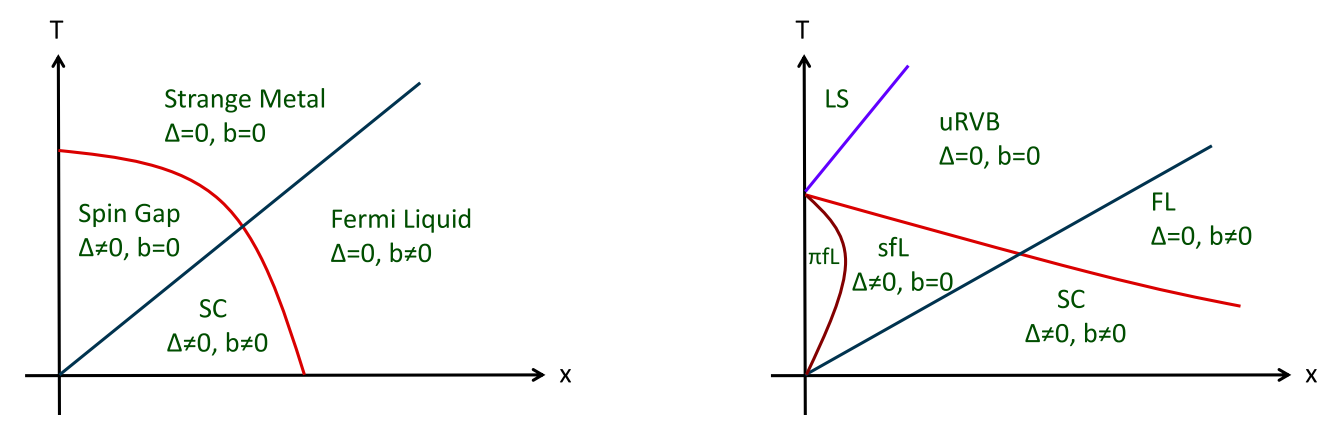

Figure 11. The mean field phase diagram as a function of temperature $T$ and doping $x$ from the $\mathrm{SU}(2)$ (left) and $\mathrm{U}(1)$ (right) slave boson theory [15]. The order parameter $\Delta$ is defined by $\Delta_{i j}=f_{i \uparrow} f_{j \downarrow}-f_{i \downarrow} f_{j \uparrow}$ and $b$ represents the expectation value of the (condensed) holon field. The $\pi$ flux phase $(\pi \mathrm{fL})$ is a spin liquid phase with the algebraic spin-spin correlation function. The localized spin (LS) phase is the phase where there are no hopping matrix elements $U_{i j}=0$ for spinons. The antiferromagnetic order is absent in the mean field approximation of the slave boson theory.

$t / J$ enters, within this approach, in determining a saddle point (mean field) and types of (emergent) gauge fluctuations around it, it does not play much role once we focus on the fluctuations around the saddle point; this can be seen, e.g., from the fact that the temporal component of the gauge field $a_{0}^{l}$ fluctuates without bound in (4.7), and its fluctuations are infinitely strong, irrespective of the value of $t / J$. In the following discussion, we focus on the transition triggered by doping (varying $\mu$ ), rather than controlling the band width (varying $t / J$ ).

At the mean field level, spinon and holons are treated as an independent entity, and are deconfined. Since the gauge coupling is (infinitely) strong, and since the monopoles that exist because of the compact nature of the gauge field could proliferate [15] we would expect there are confined phases in the phase diagram although this issue is not completely settled.

\subsection{Comparison between the holographic calculations and the slave boson approach}

The appearance of the emergent non-abelian gauge group SU(2) in the RVB theory motivates us to consider its holographic dual gravity description. Indeed, here we would like to argue that our gravity system discussed in this paper is analogous to the RVB theory of high- $T_{c}$ superconductors, though the large $N$ limit has only a qualitative relevance to our problem. ${ }^{6}$ Before we proceed to the comparison between them, we need to notice that the quantum field theory dual to our gravity system does not include massless fermions, while the RVB theory has fermionic spinons. This might be taken into account in the gravity side by introducing another (uncharged) scalar field which is dual to a fermion bilinear $\Delta$ that condenses for small $T$ and $x$. One possibility of string theory realization of this will be to add a D7-brane to our D3-brane system. ${ }^{7}$ However, here we would like to leave the further

\footnotetext{
${ }^{6}$ Refer to e.g. [31] for an analysis assuming the large $N$ limit with the gauge group $\operatorname{Sp}(N)$.

${ }^{7} \mathrm{~A}$ simple way to realize this is to wrap the D7-brane on $t, x, y, r, \chi$ and $S^{3} \in S^{5}$. It is easy to see that the fermion bilinear, which is dual to a mass deformation, condenses in this mode as the D7-brane
} 
analysis for a future work and to proceed by roughly identifying the confined phase and fermion condensed phase, as the latter is induced to the strongly coupled gauge interactions.

We first identify the confined phase (the AdS soliton phase) as a fully-gapped phase of electrons, such as the valence bond solid (VBS) phase, or the stripe phase that exists in $\mathrm{La}_{2-x} \mathrm{Ba}_{x} \mathrm{CuO}_{4}$ at $x=1 / 8$. In the slave boson theory, such a phase is a confined phase where spinons and holons are completely glued together, and all excitations as well as the ground state are well-described in terms electrons. It may also be possible to identify it with the antiferromagnetic phase in the high- $T_{c}$ cuprates, where the low-lying excitation is antiferromagnetic magnons, which is a bound state of two spinons. This gapless antiferromagnetic magnon may be interpreted as a Nambu-Goldstone boson induced by the fermion condensation.

The chemical potential $\mu$ in the gravity theory plays the similar role as doping $x$ in the cuprates; the chemical potential $\mu$ destroys the confining phase (the AdS soliton phase) just like doping $x$ frustrates the antiferromagnetic order. The phase emerges as we increase $x$ in the cuprates is superconducting phase; the corresponding phase in the gravity theory is the AdS soliton superconductor phase that emerges as we increase $\mu$. At the mean field level, the superconducting phase in the cuprates is the phase where holons are condensed. In the gravity dual, this is interpreted as the condensation of the scalar field $\Phi$. Note that in this identification we are not viewing the scalar field $\Phi$ as the Cooper pair, but a gauge invariant operator made from two holons.

It is then tempting to identify the deconfining phase (the AdS RN black hole phase), lying in the high temperature region above the AdS soliton and the AdS soliton superconductor phases, as the pseudo gap phase and the strange metal phase in high- $T_{c}$, which crossover with each other. Indeed, the AdS RN black hole phase, in the presence of fermions, shows the non-Fermi liquid behavior [32-35] due to the near horizon $\mathrm{AdS}_{2}$ geometry [34-36]. In the eariler literature [37], the relevance of AdS charged black hole was pointed out in order to construct the gravity dual of fermi surfaces. All of these are supportive for our identification of the AdS RN black hole phase as the pseudo gap or the strange metal phase, and also the AdS soliton superconductor phase as the superconductor phase of the high- $T_{c}$ cuprates.

One may notice that while the pairing symmetry of the cuprate high- $T_{c}$ superconductivity is known to be $d$-wave, there is nothing comparable to it in our gravity calculations. However, this is not a serious problem as our gravity calculation corresponds to a effective field theory limit and cannot directly distinguish between $s$-wave and $d$-wave as is so in the Ginzburg-Landau theory.

Finally, the AdS BH superconductor phase that exists for the large chemical potential can be considered as the Fermi liquid phase in the over-doped cuprates. Even though its gravity dual is a superconductor, the fermions do not condense in our approximation as it is situated at the deconfined phase. Notice that the expectation value of Cooper pair

should bend due to the bubble of nothing at $r=r_{0}$. In this case, the fermion condenses just at the confinement/deconfinement transition point. 


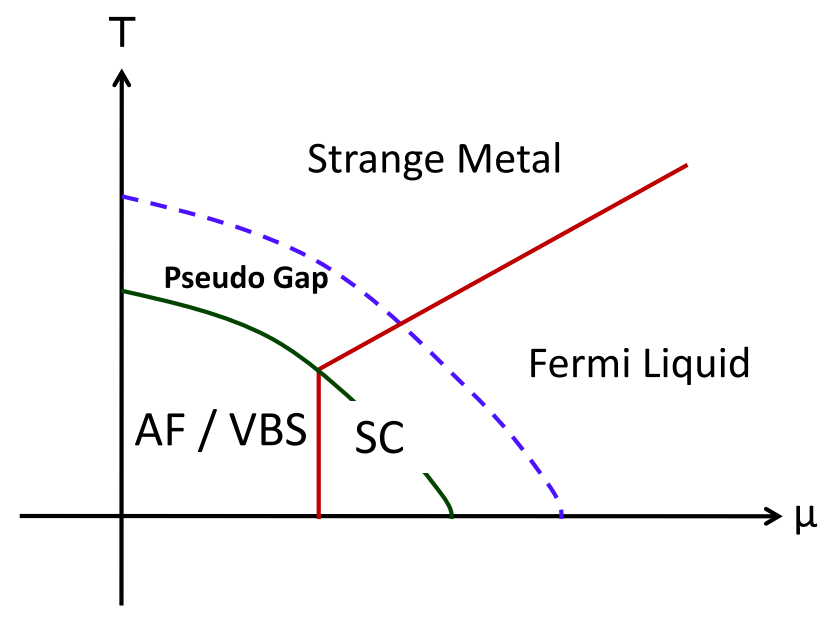

Figure 12. A speculated phase diagram of our five dimensional Einstein-Maxwell-scalar theory with an extra neutral scalar which is dual to a fermion bilinear. The newly added phase boundary which signals the fermion pair condensation is written as the dotted blue curve. The other red and green curves denote the superconductor and the deconfinement transition, respectively, as in the same way as in figure 9 .

(assuming $\left\langle b_{2 i}\right\rangle=0$ )

$$
\left\langle c_{i \uparrow} c_{j \downarrow}-c_{i \downarrow} c_{j \uparrow}\right\rangle \simeq\left\langle\Delta_{i j}\right\rangle\left\langle b_{1 i}^{\dagger}\right\rangle\left\langle b_{1 j}^{\dagger}\right\rangle,
$$

is non-vanishing iff both the fermions and holons condense. Therefore, this system from the viewpoint of electrons should not be regarded as a superconductor. Instead, it is an ordinary fermi liquid as the spin and charge are tied strongly due to the holon condensation.

Now let us compare the global phase structure of our holographic system and that of the RVB theory. At the qualitative level, they look quite similar except that our system does not actually include the massless fermions (spinons), which makes the distinction between the pseudo gap phase and the strange metal phase unclear. Even though we did not realize the fermions, it is not difficult to speculate how the phase structure of our gravity system changes, for example, by including an extra scalar field which is uncharged under the $\mathrm{U}(1)$ and is dual to the fermion bilinear. We expect that the fermion pair condensation continues slightly above the confinement/deconfinement phase boundary as in the familiar D3-D7 example [38]. This is shown in figure 12 and we filled in the corresponding phase in high- $T_{c}$.

One may notice that in hole doped high- $T_{c}$ superconductors, the antiferromagnetic phase is not exactly next to the superconductor phase (see figure 10) and the pseudo gap phase extends between them as opposed to our holographic system. In this sense, the phase diagram of electron doped high- $T_{c}$ superconductors looks more similar to ours.

\section{Conclusions}

In this paper, we study a five-dimensional Einstein-Maxwell-scalar field theory with a negative cosmological constant. We imposed that the boundary of the five-dimensional spacetime approaches a Scherk-Schwartz compactification of $\mathrm{AdS}_{5}$. The holographic dual should be a certain confining (2+1)-dimensional gauge theories with $\mathrm{U}(1)$ current, which 
includes the four-dimensional super Yang-Mills compactified on a circle. We showed that this system undergoes a phase transition into a superconductor phase (AdS soliton superconductor phase) due to the charged scalar field condensation when we increase the chemical potential at least for specific values of the mass and charge of the scalar field. We would like to leave a more extensive analysis for a future work. We argue that this is a gravity dual of superconductor/insulator phase transition at zero temperature. We also showed that we can consistently embed our phase transition into the $\mathcal{N}=1$ superconformal field theory dual to $\mathrm{AdS}_{5} \times T^{1,1}$.

We further study the global phase structure of this system by taking into account the confinement/deconfinement transition at high enough temperature. We compared our phase diagram (figure 9 ) with that of high- $T_{c}$ cuprates and we found a qualitative similarity. We also explained this fact from the viewpoint of the RVB theory of high- $T_{c}$ superconductors, where an emergent non-abelian gauge field appears due to the strong Coulomb repulsive force. This argument suggests that the AdS soliton superconductor phase is dual to the high- $T_{c}$ superconductivity phase in the RVB theory.

There are a number of future directions. First of all, since our analysis is restricted to a probe approximation, it is very interesting to perform a full analysis with backreactions. This should make clear the separation between the two superconductor phases. Also, to make the comparison with the RVB theory clear, it is important to take into account massless fermions which play the role of spinons. In order to obtain more information of the AdS soliton superconductor, it is useful to exert a magnetic flux in this system. It may also be intriguing to calculate the entanglement entropy via the AdS/CFT [39-41] to understand better the phase transition between the insulator and superconductor phase because this quantity is non-vanishing even at zero temperature (for the confinement/deconfinement phase transition, this was done in $[42,43])$. Finally, since our results depend on the values of charges of operators and their conformal dimensions, it will be useful to perform a systematic analysis in concrete string theory setups.

\section{Acknowledgments}

It is a great pleasure to thank T. Eguchi, G. Horowitz, H. Liu, Y. Ran for useful discussions. We are very grateful to Y. Hikida and S.-S. Lee for comments on the draft of this paper. TN and TT is supported by World Premier International Research Center Initiative (WPI Initiative), MEXT, Japan. The work of TN is supported by JSPS Grant-in-Aid for Scientific Research No.19.3589. The work of TT is also supported in part by JSPS Grant-in-Aid for Scientific Research No.20740132, and by JSPS Grant-in-Aid for Creative Scientific Research No. 19GS0219. SR thanks the Center for Condensed Matter Theory at University of California, Berkeley for its support.

\section{A Schrödinger potential description}

\section{A.1 AdS soliton}

The condensation of the scalar operator can be interpreted as the instability of the scalar field around the tip of the AdS soliton solution as follows. The equation of motion of the 


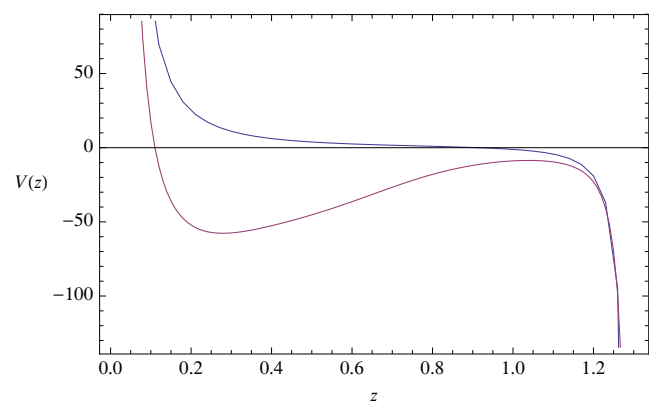

Figure 13. The potential $V(z)$ for the AdS soliton without (blue) and with (red) the scalar operator condensation.

scalar field $\Psi$ with a time dependence $e^{-i \omega t}$ gives

$$
\Psi^{\prime \prime}+\left(\frac{f^{\prime}}{f}+\frac{3}{r}\right) \Psi^{\prime}+\left(-\frac{m^{2}}{f}+\frac{\Phi^{2}+\omega^{2}}{r^{2} f}\right) \Psi=0 .
$$

This equation can be transformed to the Schrödinger equation by introducing a new radial coordinate

$$
z=\int_{r}^{\infty} \frac{d s}{s \sqrt{f(s)}}=\frac{{ }_{2} F_{1}\left(\frac{1}{4}, \frac{1}{2}, \frac{5}{4}, \frac{1}{r^{4}}\right)}{r},
$$

and redefining the scalar field like $\Psi \equiv B \psi$ with appropriate choice of the function $B$, which is given by $B(z)=r(z)^{-1 / 2}\left|r^{\prime}(z)\right|^{-1 / 2}$. The equation becomes

$$
-\partial_{z}^{2} \psi(z)+V(z) \psi=\omega^{2} \psi(z)
$$

where the potential is calculated to be

$$
V(z)=\frac{\left(15+4 m^{2}\right) r(z)^{8}-2\left(9+2 m^{2}\right) r(z)^{4}-1}{4 r(z)^{2}\left(r(z)^{4}-1\right)}-q^{2} \Phi^{2} .
$$

This potential $V(z)$ is plotted in figure 13 with and witout the scalar operator condensation, i.e., $\Phi$ takes zero and nontrivial values, respectively. The range of $z$ is $0<z \leq z_{*}$, where $z_{*}=\frac{\sqrt{\pi} \Gamma(5 / 4)}{\Gamma(3 / 4)}(\simeq 1.31)$.

We can find the following behaviors:

$$
\begin{aligned}
& V(z) \simeq \frac{m^{2}+\frac{15}{4}}{z^{2}} \quad(z \rightarrow 0), \\
& V(z) \simeq-\frac{1}{4\left(z-z_{*}\right)^{2}} \quad\left(z \rightarrow z_{*}\right) .
\end{aligned}
$$

It is well-known that the Schrödinger problem for the potential $V(z)=\frac{k}{z^{2}}$ is ill-defined (or unstable) for $k<-1 / 4$ as this leads to infinitely many negative energy states. When applied to (A.5), this leads to the BF bound $m^{2} \leq-4$. On the other hand, the behavior near the tip (A.6) shows that the system is marginally stable. In addition, the actual potential is larger than the right-hand side of (A.6) with the high potential wall near 


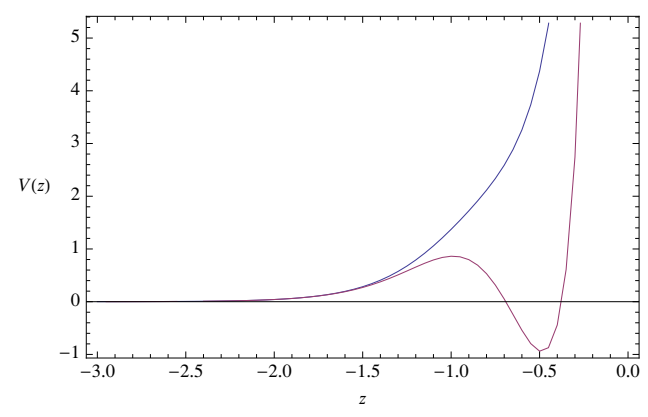

Figure 14. The potentials $V(z)$ for the AdS BH without (blue) and with (red) the scalar operator condensation.

$z=0$. Thus the ground state energy $\omega^{2}$ takes a finite positive value and this explains the mass gap in the AdS soliton. The introduction of the gauge potential $\Phi$ decreases the ground state energy and above a certain value, the system becomes unstable under the scalar condensation in the IR region.

\section{A.2 AdS black hole}

It is also useful to analyze the AdS black hole background in a similar way. We will set $\mu=0$ for simplicity. Introducing the new radial coordinate $z(-\infty<z<0)$

$$
z=-\int_{r}^{\infty} \frac{d s}{f(s)}=\frac{1}{4}\left(-\pi-2 \operatorname{coth}^{-1}(r)+2 \tanh ^{-1}(r)\right),
$$

the equation of motion for the scalar field (3.9) is simplified to the Schrödinger equation (A.3) with $B(z)=r(z)^{-3 / 2}$. The potential becomes

$$
V(z)=\frac{\left(r(z)^{4}-1\right)\left(\left(15+4 m^{2}\right) r(z)^{4}+9\right)}{4 r(z)^{6}}-q^{2} \Phi^{2} .
$$

This always vanishes on the horizon $(f=0)$ when $\Phi=0$ with arbitrary choice of $m^{2}$. The form of this potential is depicted in figure 14 with and without the scalar operator condensation.

Open Access. This article is distributed under the terms of the Creative Commons Attribution Noncommercial License which permits any noncommercial use, distribution, and reproduction in any medium, provided the original author(s) and source are credited.

\section{References}

[1] J.M. Maldacena, The large-N limit of superconformal field theories and supergravity, Adv. Theor. Math. Phys. 2 (1998) 231 [Int. J. Theor. Phys. 38 (1999) 1113] [hep-th/9711200] [SPIRES].

[2] E. Dagotto, Correlated electrons in high-temperature superconductors, Rev. Mod. Phys. 66 (1994) 763 [SPIRES].

[3] S.A. Hartnoll, C.P. Herzog and G.T. Horowitz, Building a Holographic Superconductor, Phys. Rev. Lett. 101 (2008) 031601 [arXiv:0803.3295] [SPIRES]. 
[4] S.S. Gubser, Colorful horizons with charge in anti-de Sitter space, Phys. Rev. Lett. 101 (2008) 191601 [arXiv:0803.3483] [SPIRES].

[5] C.P. Herzog, P.K. Kovtun and D.T. Son, Holographic model of superfluidity, Phys. Rev. D 79 (2009) 066002 [arXiv:0809.4870] [SPIRES].

[6] P. Basu, A. Mukherjee and H.-H. Shieh, Supercurrent: Vector Hair for an AdS Black Hole, Phys. Rev. D 79 (2009) 045010 [arXiv:0809.4494] [SPIRES].

[7] G.T. Horowitz and M.M. Roberts, Holographic Superconductors with Various Condensates, Phys. Rev. D 78 (2008) 126008 [arXiv:0810.1077] [SPIRES].

[8] S.A. Hartnoll, C.P. Herzog and G.T. Horowitz, Holographic Superconductors, JHEP 12 (2008) 015 [arXiv:0810.1563] [SPIRES].

[9] S.S. Gubser and A. Nellore, Low-temperature behavior of the Abelian Higgs model in anti-de Sitter space, JHEP 04 (2009) 008 [arXiv:0810.4554] [SPIRES].

[10] S.A. Hartnoll, Lectures on holographic methods for condensed matter physics, Class. Quant. Grav. 26 (2009) 224002 [arXiv:0903.3246] [SPIRES].

[11] C.P. Herzog, Lectures on Holographic Superfluidity and Superconductivity, J. Phys. A 42 (2009) 343001 [arXiv:0904.1975] [SPIRES].

[12] E. Witten, Anti-de Sitter space, thermal phase transition and confinement in gauge theories, Adv. Theor. Math. Phys. 2 (1998) 505 [hep-th/9803131] [SPIRES].

[13] G.T. Horowitz and R.C. Myers, The AdS/CFT Correspondence and a New Positive Energy Conjecture for General Relativity, Phys. Rev. D 59 (1998) 026005 [hep-th/9808079] [SPIRES].

[14] S.W. Hawking and D.N. Page, Thermodynamics of Black Holes in anti-de Sitter Space, Commun. Math. Phys. 87 (1983) 577 [SPIRES].

[15] P.A. Lee, N. Nagaosa and X.G. Wen, Doping a Mott insulator: Physics of high-temperature superconductivity, Rev. Mod. Phys. 78 (2006) 17 [cond-mat/0410445] [SPIRES].

[16] S. Nakamura, H. Ooguri and C.-S. Park, Gravity Dual of SPatially Modulated Phase, Phys. Rev. D 81 (2010) 044018 [arXiv:0911.0679] [SPIRES].

[17] S.S. Gubser and F.D. Rocha, The gravity dual to a quantum critical point with spontaneous symmetry breaking, Phys. Rev. Lett. 102 (2009) 061601 [arXiv:0807.1737] [SPIRES].

[18] S.S. Gubser and A. Nellore, Ground states of holographic superconductors, Phys. Rev. D 80 (2009) 105007 [arXiv: 0908.1972] [SPIRES].

[19] G.T. Horowitz and M.M. Roberts, Zero Temperature Limit of Holographic Superconductors, JHEP 11 (2009) 015 [arXiv:0908.3677] [SPIRES].

[20] R.A. Konoplya and A. Zhidenko, Holographic conductivity of zero temperature superconductors, Phys. Lett. B 686 (2010) 199 [arXiv:0909.2138] [SPIRES].

[21] M. Fujita, W. Li, S. Ryu and T. Takayanagi, Fractional Quantum Hall Effect via Holography: Chern-Simons, Edge States and Hierarchy, JHEP 06 (2009) 066 [arXiv:0901.0924] [SPIRES].

[22] T. Azeyanagi, W. Li and T. Takayanagi, On String Theory Duals of Lifshitz-like Fixed Points, JHEP 06 (2009) 084 [arXiv: 0905. 0688] [SPIRES].

[23] I.R. Klebanov and E. Witten, Superconformal field theory on threebranes at a Calabi-Yau singularity, Nucl. Phys. B 536 (1998) 199 [hep-th/9807080] [SPIRES]. 
[24] S.S. Gubser, C.P. Herzog, S.S. Pufu and T. Tesileanu, Superconductors from Superstrings, Phys. Rev. Lett. 103 (2009) 141601 [arXiv:0907.3510] [SPIRES].

[25] D.J. Scalapino, E. Loh and J.E. Hirsch, d-wave pairing near a spin-density-wave instability, Phys. Rev. B 34 (1986) 8190.

[26] K. Miyake, S. Schmitt-Rink and C.M. Varma, Spin-fluctuation-mediated even-parity pairing in heavy-fermion superconductors, Phys. Rev. B 34 (1986) 6554.

[27] P. Monthoux and D. Pines, YBa2Cu3O\%: A nearly antiferromagnetic Fermi liquid, Phys. Rev. B 47 (1993) 6069.

[28] T. Moriya and K. Ueda, Spin Fluctuations and High Temperature Superconductivity, Adv. Phys. 49 (2000) 555.

[29] S.A. Hartnoll, P.K. Kovtun, M. Muller and S. Sachdev, Theory of the Nernst effect near quantum phase transitions in condensed matter and in dyonic black holes, Phys. Rev. B 76 (2007) 144502 [arXiv:0706.3215] [SPIRES].

[30] F. Denef, S.A. Hartnoll and S. Sachdev, Quantum oscillations and black hole ringing, Phys. Rev. D 80 (2009) 126016 [arXiv:0908.1788] [SPIRES].

[31] S. Sachdev and N. Read, Large $N$ expansion for frustrated and doped quantum antiferromagnets, Int. J. Mod. Phys. B 5 (1991) 219 [cond-mat/0402109].

[32] S.-S. Lee, A Non-Fermi Liquid from a Charged Black Hole: A CriticalFermi Ball, Phys. Rev. D 79 (2009) 086006 [arXiv:0809.3402] [SPIRES].

[33] M. Cubrovic, J. Zaanen and K. Schalm, String Theory, Quantum Phase Transitions and the Emergent Fermi-Liquid, Science 325 (2009) 439 [arXiv: 0904.1993] [SPIRES].

[34] H. Liu, J. McGreevy and D. Vegh, Non-Fermi liquids from holography, arXiv:0903.2477 [SPIRES].

[35] T. Faulkner, H. Liu, J. McGreevy and D. Vegh, Emergent quantum criticality,Fermi surfaces and AdS2, arXiv:0907.2694 [SPIRES].

[36] C.P. Herzog, I.R. Klebanov, S.S. Pufu and T. Tesileanu, Emergent Quantum Near-Criticality from Baryonic Black Branes, JHEP 03 (2010) 093 [arXiv:0911.0400] [SPIRES].

[37] S.-J. Rey, String theory on thin semiconductors: Holographic realization ofFermi points and surfaces, Prog. Theor. Phys. Suppl. 177 (2009) 128 [arXiv:0911.5295] [SPIRES].

[38] S. Kobayashi, D. Mateos, S. Matsuura, R.C. Myers and R.M. Thomson, Holographic phase transitions at finite baryon density, JHEP 02 (2007) 016 [hep-th/0611099] [SPIRES].

[39] S. Ryu and T. Takayanagi, Holographic derivation of entanglement entropy from AdS/CFT, Phys. Rev. Lett. 96 (2006) 181602 [hep-th/0603001] [SPIRES].

[40] S. Ryu and T. Takayanagi, Aspects of holographic entanglement entropy, JHEP 08 (2006) 045 [hep-th/0605073] [SPIRES].

[41] T. Nishioka, S. Ryu and T. Takayanagi, Holographic Entanglement Entropy: An Overview, J. Phys. A 42 (2009) 504008 [arXiv:0905.0932] [SPIRES].

[42] T. Nishioka and T. Takayanagi, AdS bubbles, entropy and closed string tachyons, JHEP 01 (2007) 090 [hep-th/0611035] [SPIRES].

[43] I.R. Klebanov, D. Kutasov and A. Murugan, Entanglement as a Probe of Confinement, Nucl. Phys. B 796 (2008) 274 [arXiv:0709.2140] [SPIRES]. 